\title{
THE CONCEPTUAL DESIGN OF A NEW TRANSFER LINE FROM BOOSTER TO RECYCLER FOR THE FERMILAB PROTON PLAN PHASE 2 CAMPAIGN*
}

\author{
D. E. Johnson ${ }^{\#}$, M. Xiao, FNAL, Batavia, IL , U.S.A
}

\begin{abstract}
Upon the termination of the Fermilab Collider program, the current Recycler anti-proton storage ring (RR) will be converted to a proton pre-injector for the Main Injector (MI) synchrotron. This is scheduled to increase the beam power for the $120 \mathrm{GeV}$ Neutrino program to upwards of $700 \mathrm{KW}$. A transport line that can provide direct injection from the Booster to the Recycler while preserving direct injection from the Booster into the Main Injector and the $8 \mathrm{GeV}$ Booster Neutrino program will be discussed, and its concept design will be presented.
\end{abstract}

\section{INTRODUCTION}

The Recycler is being modified from a anti-proton storage ring to a proton pre-injector for the MI as part of the Nova Project. This requires direct injection from Booster into the RR. An "upper $8 \mathrm{GeV}$ line" was envisioned at the time of the Recycler design and at least one utility, an LCW header, was positioned at the junction of the $8 \mathrm{GeV}$ line tunnel and the MI to allow such a line to exist. Subsequent to the construction of the MI, an $8 \mathrm{GeV}$ neutrino experiment, mini-Boone, was installed north of the MI10 injection area. This experiment utilizes $15 \mathrm{~Hz}$ Booster beam pulses not injected into the MI. A switch magnet and transport line to their target was installed at the end of the $8 \mathrm{GeV}$ line, just downstream of the $8 \mathrm{GeV}$ line quad, Q851, which selects beam for the experiment

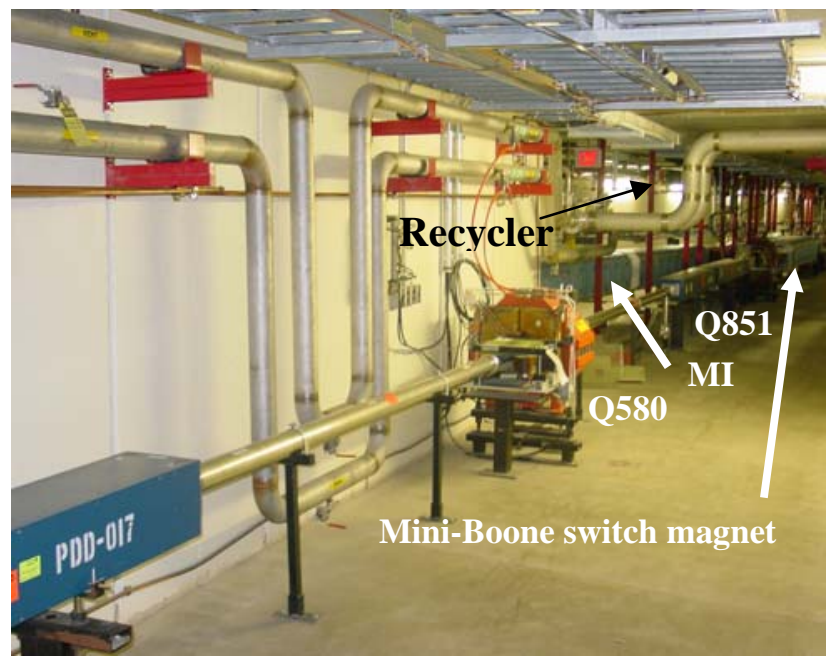

Figure 1: Picture of the end of the $8 \mathrm{GeV}$ line where it meets the Main Injector

*Operated by Fermi Research Alliance, LLC under contract No. DE-AC02-07CH11359 with the United States Department of Energy.

\#dej@fnal.gov when energized.

Figure 1 shows a picture of the interface of the $8 \mathrm{GeV}$ line, the MI tunnel and the mini-Boone switch magnet. It can be seen that this region of the tunnel is a "wide-span" tunnel and become extremely crowded the farther downstream one goes.

\section{DESCRIPTION}

The MI and Recycler follow the approximate same footprint, but are separated vertically by 56 inches. The elevation of the existing $8 \mathrm{GeV}$ line into the MI is just shy of 2 inches higher than the MI. There are two generic layouts for creating a vertical "dog-leg" displacement. One requires only two bending centers (one-up and onedown) separated by in phase by 360 degrees (if beta's are equal at the dipoles). The other requires four bend centers, where the beam is bent up, leveled off, bent up again, and leveled off at the desired elevation. If the pair of up bends are separated by 180 degrees and the pair of down bends are separated by 180 degrees, and the beta's are equal then we get perfect cancellation of the vertical dispersion. This second technique requires 270 degrees of phase if each bend is 90 degrees apart. This second technique is currently used in the MI to RR injection/extraction transport lines. The current solution is a hybrid between these two methods. It uses three vertical bending centers. A cartoon of the transfer line is shown in Figure 2. The first vertical bend (V1 switch) bends up at about $33 \mathrm{mr}$ angle to clear downstream magnets. Once enough elevation has been reached to clear the lower $8 \mathrm{GeV}$ line beam pipe and next magnet, a vertical dipole (V2, rotated PDD permanent magnet) reduces the pitch from $33 \mathrm{mr}$ to about $14 \mathrm{mr}$. These two bends act as a single extended bend center. This pitch is maintained until the third vertical bending center, the MLAW injection Lambertson located just upstream of Q102A, where it is placed on the correct vertical closed orbit of the Recycler and cancels the vertical dispersion created by the switch magnet.

\section{LATTICE}

The Recycler injection transport line segment can be described as a FODO lattice with a vertical achromat at the end of a pre-existing horizontal achromat. The transport line is composed of all permanent magnets with the exception of the vertical switch magnet, the vertical Lambertson, and powered trim magnets. Figure 3 shows the lattice and dispersion functions for matching into the Recycler. 


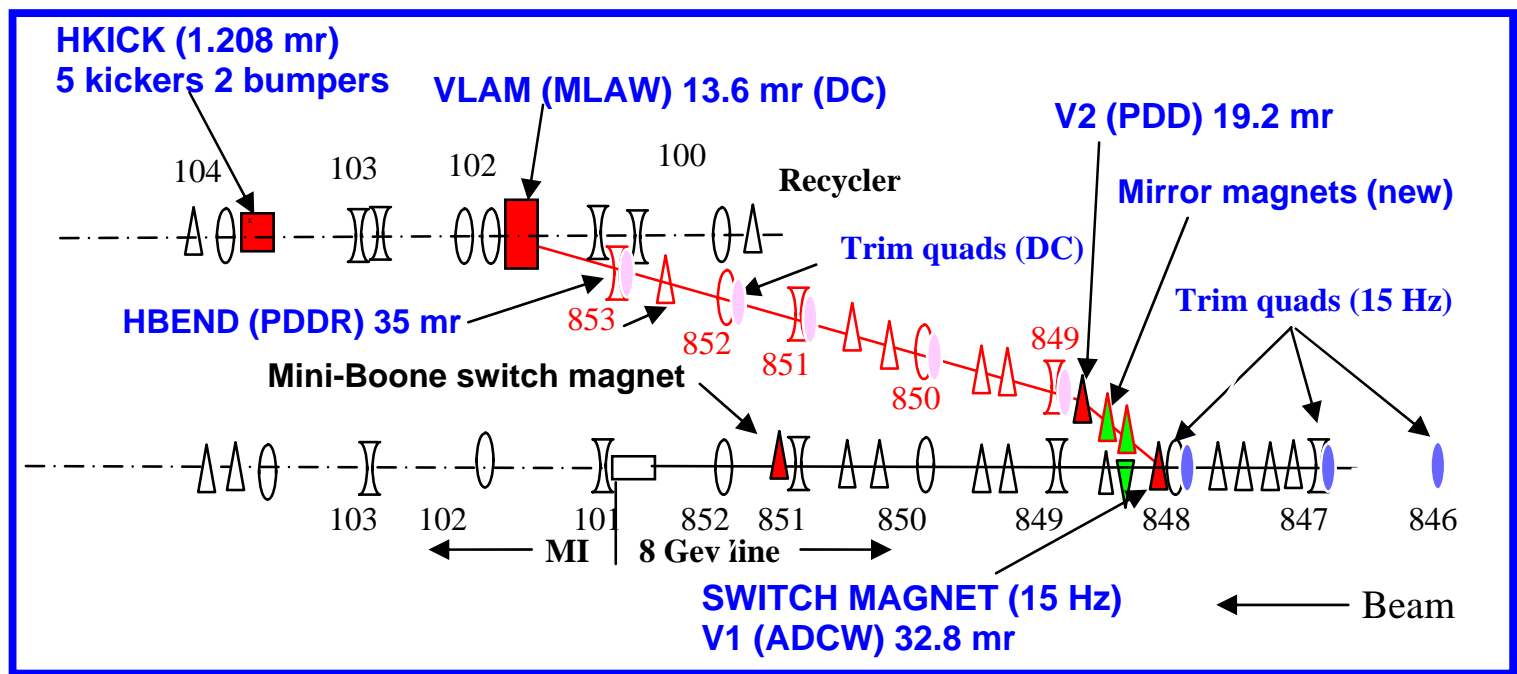

Figure 2: Cartoon of the new Booster to Recycler transport line shown in red. Also shown are a downstream segment of the existing $8 \mathrm{GeV}$ line, the Main Injector, and Recycler. The items labeled in blue text are new and items in black text are existing.

\section{MATCHING}

The transport line must be able to match the eight optical functions in the RR and, when the switch magnet is off, match into the MI and the mini-Boon Neutrino line. To this end, the gradients at the locations 846 thru 848 in the existing $8 \mathrm{GeV}$ line and gradients at the locations 849 thru 853 in the new segment are adjusted to match into

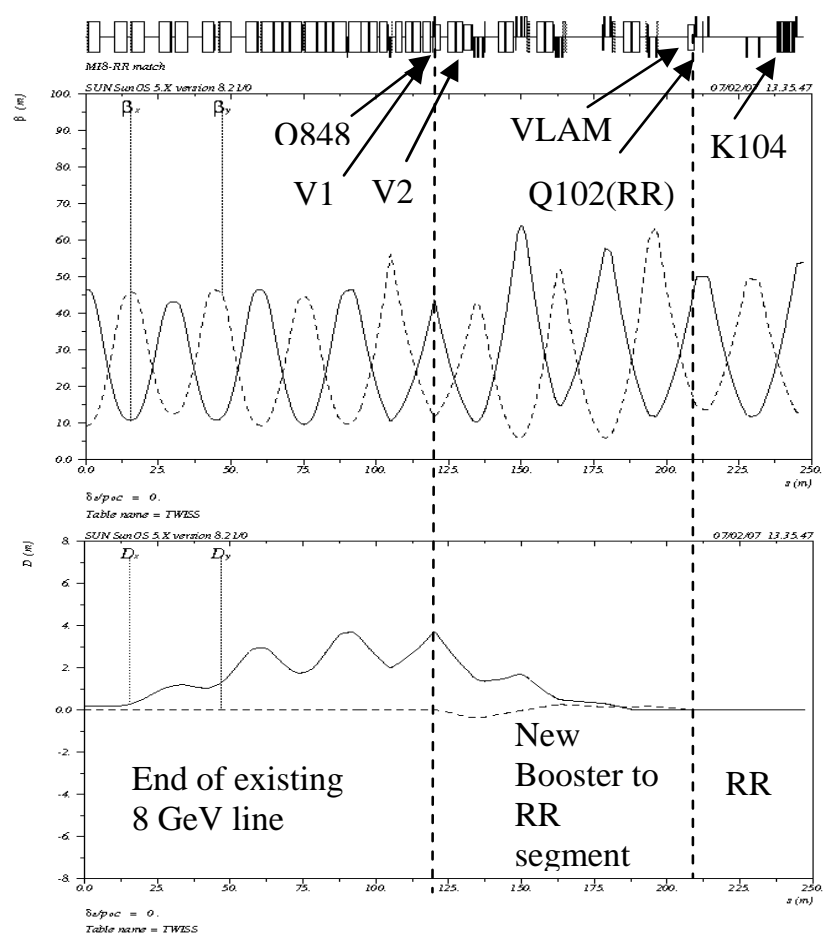

Figure 3: Lattice and dispersion functions of a segment of the existing $8 \mathrm{GeV}$ line, the new Booster to Recycler segment, and the Recycler.

the Recycler.
A trim quad will be added to the current $8 \mathrm{GeV}$ line at the locations of Q846 thru Q848 which can be utilized at $15 \mathrm{~Hz}$ for compensating differences in matching gradients into the Recycler, MI and/or mini-Boone.

\section{RECYCLER INJECTION}

The injection into the Recycler uses a standard vertical Lambertson with a horizontal kicker located $90^{\circ}$ downstream to place the trajectory on the horizontal closed orbit. A modified version of the existing injection Lambertson will be used in the Recycler and it will be installed just upstream of Q102A. The bend field gap will be increased by about $14 \%$ to $50 \mathrm{~mm}$. The required strength is $40 \%$ of the current installation in the MI, so any saturation leakage field should be minimized. This Lambertson will run DC. The Lambertson is rolled $\sim 5^{\circ}$ toward the wall. The strength requirement on the kickers is directly related to the injected an circulating beam separation at the injection Lambertson. The required angle for closure is $1.208 \mathrm{mr}$. The rise time requirement is determined by the batch separation in the Recycler. Currently, we are expecting to inject 81 bunches out of a possible 84 (Booster circumference) which leaves 57 ns between batches for the kicker rise and fall time. This implies multiple short kicker modules. The current solution uses 5 modules each with an integrated field of $71.6 \mathrm{kG}-\mathrm{m}$ (75 kG-m max). The kicker group will be installed immediately upstream of Q104A.

\section{EMITTANCE GROWTH}

There are three main sources of emittance growth due to a mismatch between a transport line and ring in the absence of any vacuum windows. These are due to amplitude function mismatch, dispersion mismatch, and steering errors. Although the gradients of the permanent 
magnet quads and the dipole fields of the permanent dipoles are determined for a perfect match, trim quads and trim dipoles are installed at each quad location for fine tuning the amplitude function match and orbit control/closure (steering errors). Although the dispersion match is primarily determined by the design geometry and design phase advance between bend centers, the trim quads will only have minor impact on the dispersion match.

We specify that the emittance growth due to all three mechanisms should be less that $1 \pi$-mm-mr for an incoming 95\% normalized emittance of $25 \pi$-mm-mr (4\%) then we can estimate tolerances on matching to the Recycler. Assuming equal contributions, this translates to an emittance growth of less than $0.577 \pi$ for any one contribution.

The emittance growth due to an amplitude function mismatch is proportional to the incoming emittance. In the absence of a error in the slope of the amplitude function, an error of $25 \%$ in beta will lead to a $2.5 \%$ increase in emittance $(0.0625 \pi)$ and in the absence of an amplitude function mismatch, a factor or 3 error in the slope (alpha) will only lead to a $2 \%$ increase in emittance.

A dispersion mismatch is proportional to the momentum spread. For an energy spread $\Delta \mathrm{p} / \mathrm{p}$ of $0.12 \%$, the $\sigma \mathrm{p} / \mathrm{p}$ for a gaussian distribution is about $0.5 \mathrm{E}-3$. Given this, for $8 \mathrm{GeV}$ injection, a 1.1 meter dispersion mismatch is required to produce a $0.6 \pi$ increase in vertical emittance. In the horizontal plane, the beta and alpha at the center of the kickers are about 50m and -2.6. Again, assuming the slope of the dispersion is matched, it would require 0.75 meter dispersion mismatch to produce the same $0.6 \pi$ growth.

To estimate the sensitivity of injection closure in each plane, we calculate the magnitude of position error, assuming no injection angle error, that would produce about a $0.6 \pi$ growth. This corresponds to a tolerance of $0.4 \mathrm{~mm}$ position error for both the horizontal and vertical planes. It is assumed that the existing Recycler injection closure program will be adapted for this injection. Current closure into the Recycler from MI is typically better than $0.2 \mathrm{~mm}$ after 1 or 2 iterations.

\section{MAIN INJECTOR/MINI-BOONE MATCHING}

Matching into the MI requires adjustment of the three electromagnet trim quads at 846-848, and the four existing $8 \mathrm{GeV}$ line electromagnet quads at 849 to 852 . Here, there is no vertical dispersion match, only transverse lattice functions and horizontal dispersion. To match the mini-Boone line we utilize the constraint on beta and alpha at the entrance into the transport line (i.e. the exit of Q851) at the same time we match into the MI, dropping the matching constraint on the horizontal dispersion. All gradients are within limits for specific quad types. Figure 4 shows the solution to both MI and mini-Boone optics.

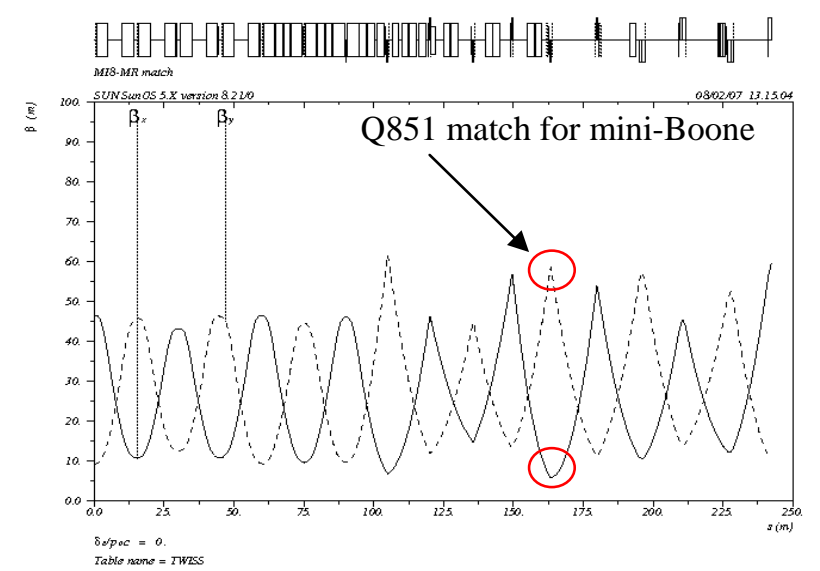

Figure 4: Lattice function match to mini-Boone and the Main Injector.

\section{APERTURE}

The transfer line physical aperture must be large enough to cleanly transport the nominal beam from Booster with additional room for closed orbit manipulation. For the calculation of a beam envelope, we assume a transverse emittance of $25 \pi-\mathrm{mm}-\mathrm{mr}$ (conservative) and a momentum spread, $\Delta \mathrm{p} / \mathrm{p}$ of $0.24 \%$ (very conservative, factor of 2). We calculate a $95 \%$ normalized beam sigma by

$$
\sigma={\sqrt{\frac{\beta \varepsilon}{6(\beta \gamma)}+D^{2}\left(\frac{\sigma_{p}}{p}\right)^{2}}}^{2}
$$

where $\sigma \mathrm{p} / \mathrm{p}=(1 / \mathrm{sqrt}(6)) \Delta \mathrm{p} / \mathrm{p}$ and $\beta \gamma$ is 9.47. Using this, $+/-2.45 \sigma$ contains $95 \%$ of the beam. We then take 10 times this sigma to account for beam halo and steering. The physical apertures are verified to be at least 10 sigma.

\section{SUMMARY}

The modifications to the Recycler and the injection/extraction lines have moved beyond the Proton Plan and have now been incorporated into the Nova project as discussed. The next phase of the design will include detailed vacuum engineering and mechanical design. Plans are in place to "stake out" the lines and verify any interferences during the 2007 Accelerator Shutdown. Further information may be obtained from documents listed in reference [1].

\section{REFERENCES}

[1] D. E. Johnson, Nova database documents, http://novadocdb.fnal.gov/cgi-bin/ListBy?authorid=224 
